\title{
Economic burden of bronchiectasis in Germany
}

\author{
Roland Diel (1) ${ }^{1,2,3}$, James D. Chalmers ${ }^{4}$, Klaus F. Rabe ${ }^{2}$, Albert Nienhaus ${ }^{3,5}$, \\ Robert Loddenkemper ${ }^{6}$ and Felix C. Ringshausen ${ }^{7}$
}

Affiliations: ${ }^{1}$ Institute for Epidemiology, University Medical Hospital Schleswig-Holstein, Kiel, Germany. ${ }^{2}$ Lung Clinic Grosshansdorf, Airway Research Center North (ARCN), German Center for Lung Research (DZL), Grosshansdorf, Germany. ${ }^{3}$ Institution for Statutory Accident Insurance and Prevention in the Health and Welfare Services (BGW), Hamburg, Germany. ${ }^{4}$ Scottish Centre for Respiratory Research, University of Dundee, Ninewells Hospital and Medical School, Dundee, UK. ${ }^{5}$ Institute for Health Service Research in Dermatology and Nursing, University Medical Center Hamburg-Eppendorf, Hamburg, Germany. ${ }^{6}$ German Central Committee against Tuberculosis, Berlin, Germany. ${ }^{7}$ Dept of Respiratory Medicine, Hannover Medical School and Biomedical Research in End-stage and Obstructive Lung disease (BREATH), German Center for Lung Research (DZL), Hannover, Germany.

\section{Correspondence:}

Roland Diel, Institute of Epidemiology, University Hospital Schleswig-Holstein, Campus Kiel, Niemannsweg 11, 24105 Kiel, Germany.

E-mail: roland.dieldepi.uni-kiel.de

@ERSpublications

Newly diagnosed bronchiectasis causes substantial healthcare costs and has high mortality http://ow.ly/ $\mathrm{EHtO} 30 \mathrm{mUaPf}$

Cite this article as: Diel R, Chalmers JD, Rabe KF, et al. Economic burden of bronchiectasis in Germany. Eur Respir J 2019; 53: 1802033 [https://doi.org/10.1183/13993003.02033-2018].

ABSTRACT Estimates of healthcare costs for incident bronchiectasis patients are currently not available for any European country.

Out of a sample of 4859013 persons covered by German statutory health insurance companies, 231 new bronchiectasis patients were identified in 2012. They were matched with 685 control patients by age, sex and Charlson Comorbidity Index, and followed for 3 years.

The total direct expenditure during that period per insured bronchiectasis patient was EUR18634.57 (95\% CI EUR15891.02-23871.12), nearly one-third higher (ratio of mean 1.31, 95\% CI 1.02-1.68) than for a matched control $(\mathrm{p}<0.001)$. Hospitalisation costs contributed to $35 \%$ of the total and were $>50 \%$ higher in the bronchiectasis group (ratio of mean 1.56, 95\% CI 1.20-3.01; p<0.001); on average, bronchiectasis patients spent 4.9 (95\% CI 2.27-7.43) more days in hospital ( $\mathrm{p}<0.001)$. Antibiotics expenditures per bronchiectasis outpatient (EUR413.81) were nearly 5 times higher than those for a matched control (ratio of mean 4.85, 95\% CI 2.72-8.64). Each bronchiectasis patient had on average 40.5 (95\% CI 17.1-43.5) sick-leave days and induced work-loss costs of EUR4230.49 (95\% CI EUR2849.585611.20). The mortality rate for bronchiectasis and matched non-bronchiectasis patients after 3 years of follow-up was $26.4 \%$ and $10.5 \%$, respectively $(\mathrm{p}<0.001)$. Mortality in the bronchiectasis group was higher among those who also had chronic obstructive lung disease than in patients with bronchiectasis alone (35.9\% and $14.6 \%$, respectively; $\mathrm{p}<0.001)$.

Although bronchiectasis is considered underdiagnosed, the mortality and associated financial burden in Germany are substantial. 


\section{Introduction}

Bronchiectasis is characterised by irreversibly dilated airways leading to chronic bacterial infection, which leads to persistent productive cough, haemoptysis, shortness of breath and chronic fatigue. Patients also experience episodes of exacerbations [1]. Importantly, chronically infected patients, especially those infected with Pseudomonas aeruginosa, may have reduced quality of life, require more frequent hospitalisations and carry significantly higher risk of death [2].

Pulmonary diseases other than cystic fibrosis (CF) may predispose patients to bronchiectasis, which is then referred to as non-CF bronchiectasis [3]. Although no underlying conditions can be identified in up to $32-50 \%$ of bronchiectasis cases [3, 4], recognised causes include lung infection by nontuberculous mycobacteria [5], connective tissue diseases $[6,7]$ and allergic bronchopulmonary aspergillosis [8]. Many patients with bronchiectasis will initially be diagnosed with chronic airflow limitation [9] and thus may be misdiagnosed as chronic obstructive lung disease (COPD). However, bronchiectasis may be a secondary complication of asthma and COPD: as recently described by Quint et al. [10], 42.5\% of bronchiectasis patients in the UK had a coexisting diagnosis of asthma and $36.1 \%$ had a coexisting diagnosis of COPD.

Although bronchiectasis has previously been classified as an "orphan disease", its incidence is now considered to be increasing worldwide $[10,11]$. As more patients require appropriate management, it is necessary to assess the resource requirements for the treatment of bronchiectasis within the various European healthcare systems. However, estimates of healthcare costs for newly diagnosed bronchiectasis patients are currently not available for any European country. Therefore, the objective of the current study was to estimate the burden of illness associated with incident bronchiectasis in Germany.

\section{Methods}

\section{Setting and data collection}

This study was a population-based cohort study with a nested case-control design, based on administrative data from statutory company health insurance funds from all regions of Germany. The data were provided by the database service provider sgh consulting (Hamburg, Germany; www.sgh-consulting.de). The health claims database of that company comprises anonymised billing data from longitudinally linked records of 4850013 insured persons (effective date: July 31, 2017), and is representative with respect to age and sex for the around 70 million Germans mandatorily insured under the statutory company health insurance scheme. Patients with bronchiectasis were identified based on the 10th revision of the International Classification of Diseases (German modification) (ICD-10 GM) code J47. Patients classified as having newly diagnosed bronchiectasis were identified in 2012 and tracked over 3 years after the first (index) quarter in which $\mathrm{J} 47$ was coded.

Patients were classified as having incidental bronchiectasis if they met the following criteria: 1) at least one medical claim with a documented ICD-10 GM code J47 as an inpatient or with a verified diagnosis as an outpatient and 2) no documented ICD-10 GM code J47 in the eight quarters prior to the index quarter in 2012 (figure 1). Patients with bronchiectasis who had either an ICD-10 GM diagnosis code E84.- (CF) or Q33.4 (congenital bronchiectasis) were excluded.

To calculate the increase in healthcare costs attributable to the presence of bronchiectasis, control patients without bronchiectasis were randomly assigned an index quarter in 2012 according to the distribution of index quarters in bronchiectasis patients. These were followed for a total period of 3 years without limitation to a calendar year or until death. The control group was matched to the group of bronchiectasis patients at a ratio of 3:1 with regard to age, sex, and in terms of their distribution and level of comorbidities. Therefore, patients' comorbidities were assessed and measured using the Charlson Comorbidity Index (CCI) score, a claims-based measure of overall disease burden based on the occurrence of at least one of 17 comorbidities identified using the ICD-10 GM coding manual $[12,13]$, and the frequencies of comorbidity patterns of the matched non-bronchiectasis sample were as closely related as possible to those of the bronchiectasis patients.

As some diseases are known to be extraordinary cost drivers and therefore may bias the results of the study, bronchiectasis patients as well as matched control patients were excluded a priori if they suffered from one of the diseases that exceeded EUR20000 in allocation according to the German morbidity-based risk structure equalisation ("high-cost cases") [14].

Costs over the entire follow-up period were retrieved for six different categories: inpatient care (hospitalisation), outpatient visits and diagnostics, prescribed pharmaceuticals according to German national drug (Anatomical Therapeutic Chemical (ATC)) codes, remedies (physiotherapy treatments and modalities, such as active cycle of breathing and postural drainage techniques), medical aids (especially nebulisers and respiration therapy equipment), and sick pay. Furthermore, the top 20 prescribed drugs for bronchiectasis management were analysed and compared with those of the non-bronchiectasis group. 
Total observation period

(January 1, 2012 to December 31, 2015)

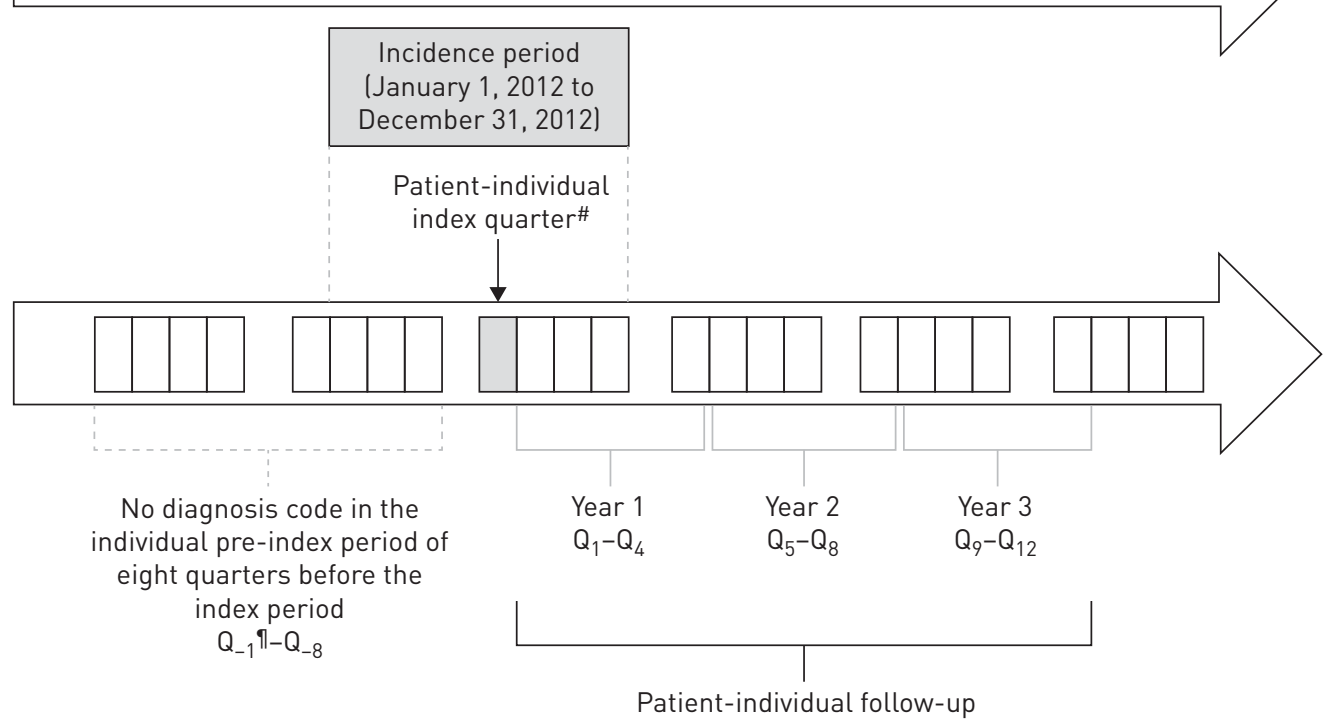

FIGURE 1 Patient-individual observation periods. \#: example of a quarter of the first bronchiectasis diagnosis in 2012 lonly verified diagnoses are considered in the outpatient setting, whereas all types of diagnoses (primary and secondary) are considered in the inpatient setting); ": all quarterly information refers to the patient-individual follow-up period relative to the index quarter.

\section{Calculation of loss of productivity due to bronchiectasis (indirect costs)}

In accordance with the human capital approach, indirect costs refer to the productivity loss for the national economy caused by absence from the workplace on sick leave.

According to the German Hanover Consensus [15], the per-day average productivity loss figure used to cover self-employed income as well is calculated as: sick-leave days $\times$ (gross income from non-self-employed work for the respective year/365 days).

The follow-up time period after diagnosis of a bronchiectasis patient is a maximum of 36 months. As per-day employer cost for employee compensation in Germany increased slightly but continuously from 2012 (2012: EUR101.19; 2013: EUR103.28; 2014: EUR106.18; 2015: EUR108.74 [16]) our assessment considered the exact calendar year within which the observation period of an individual bronchiectasis patient or a matched control was on sick leave. We multiplied the amount of per-day employer costs of a given year between 2012 and 2015 with the number of lost working days of the patients in the respective year to arrive at our total amounts.

\section{Statistical analysis}

Frequency and percentage were reported for categorical variables. For descriptive analyses, mean, standard deviation, 95\% confidence interval and median were reported for all continuous variables. To compare bronchiectasis patients and matched controls, appropriate univariate tests (i.e. the Chi-squared test of independence for categorical variables and the Wilcoxon-Mann-Whitney test for continuous variables) were used. Differences were considered significant if the two-sided p-value was $<0.05$. Ratios of the mean (RoM), including 95\% confidence intervals, were calculated for continuous outcomes.

Mortality was determined separately between both groups yearly and at the end of the study period. We generated a Kaplan-Meier survival plot and used a log-rank test to prove whether the difference in survival times between the bronchiectasis and the non-bronchiectasis group was statistically significant. Cox regression, adjusted to group membership (bronchiectasis or non-bronchiectasis), age and presence of COPD as comorbidity, was employed to calculate the hazard ratio of all-cause mortality.

All costs are reported in EUR in 2012 and the respective follow-up years in which they were incurred.

\section{Results}

Patient characteristics

After exclusion of four "high-cost" cases (two out of the bronchiectasis group and two out of the matched control group), a total of 231 bronchiectasis cases and 685 matched controls met the study criteria and 
TABLE 1 Most frequent diseases out of the range of Charlson Comorbidity Index comorbid conditions in bronchiectasis and matched control patients ${ }^{\#}$

\begin{tabular}{lcccc}
$\begin{array}{c}\text { ICD-10 GM } \\
\text { code }\end{array}$ & Description & $\begin{array}{c}\text { Bronchiectasis } \\
\text { patients }\end{array}$ & $\begin{array}{c}\text { Matched } \\
\text { controls }\end{array}$ & p-value \\
\hline J44.- & COPD & $128(55.4)$ & $246(35.9)$ & $<0.001$ \\
J45.- & Asthma & $65(28.1)$ & $185(27.0)$ & $0.78^{\text {NS }}$ \\
K21.- & Gastro-oesophageal reflux disease & $34(14.7)$ & $101(14.7)$ & $0.78^{\text {NS }}$ \\
M06.- & Other chronic polyarthritis & $6(2.6)$ & $17(2.5)$ & $1.0^{\text {NS }}$ \\
I25.- & Coronary heart disease & $36(15.6)$ & $105(15.3)$ & $0.77^{\text {NS }}$ \\
I50.- & Heart failure & $27(11.7)$ & $69(10.1)$ & $0.77^{\text {NS }}$ \\
\hline
\end{tabular}

Data are presented as $\mathrm{n}(\%)$, unless otherwise stated. COPD: chronic obstructive lung disease; ICD-10 GM: 10th revision of the International Classification of Diseases (German modification). \# : multiple entries are possible. NS: nonsignificant.

were included in the analysis. The mean age of bronchiectasis patients and matched controls was 58.6 (95\% CI 56.3-60.9) and 58.5 (95\% CI 57.2-59.8) years, respectively. 61.5\% of the patients were male and $38.5 \%$ were female. Mean CCI total score was equally high among both groups: 2.70 (95\% CI 2.4-3.0) in the bronchiectasis group and 2.76 (95\% CI 2.6-2.9) in the matched control group.

With respect to the most frequently represented diseases out of the range of predefined comorbid conditions in the CCI, more bronchiectasis patients (128 out of $231(55.4 \%)$ ) than matched controls had the diagnosis COPD ( J44.-) (table 1). Nevertheless, COPD was also the most frequent comorbid condition (246 out of $685(35.9 \%))$ in the matched control group. There were no significant differences between the two groups in the frequency of asthma (J45.-; 28.1\% of the bronchiectasis patients and $27.0 \%$ of the matched controls) and frequency of gastro-oesophageal reflux disease (K21.-; 14.7\% of all cases in both groups). Coronary heart disease (I25.-) was observed in $15.6 \%$ and $15.3 \%$, respectively, and heart failure (I50.-) in $11.7 \%$ and $10.1 \%$, respectively.

\section{Incidence of bronchiectasis}

The age- and sex-adjusted incidence rate for bronchiectasis in 2012 was 6.1 (95\% CI 6.0-6.3) per 100000 insured German inhabitants (table 2), whereby males aged $>65$ years contributed mostly with an incidence of 21.68 (95\% CI 20.57-22.86) per 100000 persons of that age group.

\section{Direct costs}

For patients with bronchiectasis, the total direct expenditure over the follow-up period per insured patient was EUR18634.57 (95\% CI EUR15891.02-23871.12), which was nearly one-third (RoM 1.31, 95\% CI

\begin{tabular}{|c|c|c|}
\hline & Patients n & Incidence per $100000^{\#}(95 \% \mathrm{Cl})$ \\
\hline \multicolumn{3}{|l|}{ Female } \\
\hline$<15$ years & 0 & $0.00(0.00-0.00)$ \\
\hline $15-65$ years & 863 & $3.66(3.42-3.91)$ \\
\hline$>65$ years & 1036 & $11.38(10.71-12.10)$ \\
\hline Total & 1899 & $5.13(4.91-5.37)$ \\
\hline \multicolumn{3}{|l|}{ Male } \\
\hline$<15$ years & 124 & $2.74(2.28-3.27)$ \\
\hline $15-65$ years & 903 & $4.03(3.78-4.31)$ \\
\hline$>65$ years & 1390 & $21.68(20.57-22.86)$ \\
\hline Total & 2417 & 7.25 (6.97-7.55) \\
\hline \multicolumn{3}{|l|}{ Total } \\
\hline$<15$ years & 124 & $1.40(1.17-1.68)$ \\
\hline $15-65$ years & 1766 & $3.84(3.66-4.03)$ \\
\hline$>65$ years & 2426 & $15.64(15.03-16.28)$ \\
\hline Total & 4316 & $6.14(5.96-6.32)$ \\
\hline
\end{tabular}

Incidence rate refers to a population of 4850013 insured persons who could be followed for the entire period of 36 months. " : per 100000 persons under risk (insured persons). 
TABLE 3 Total cost of bronchiectasis and costs separated by main cost types compared with matched control patients after adjusting for age, sex and Charlson Comorbidity Index scores

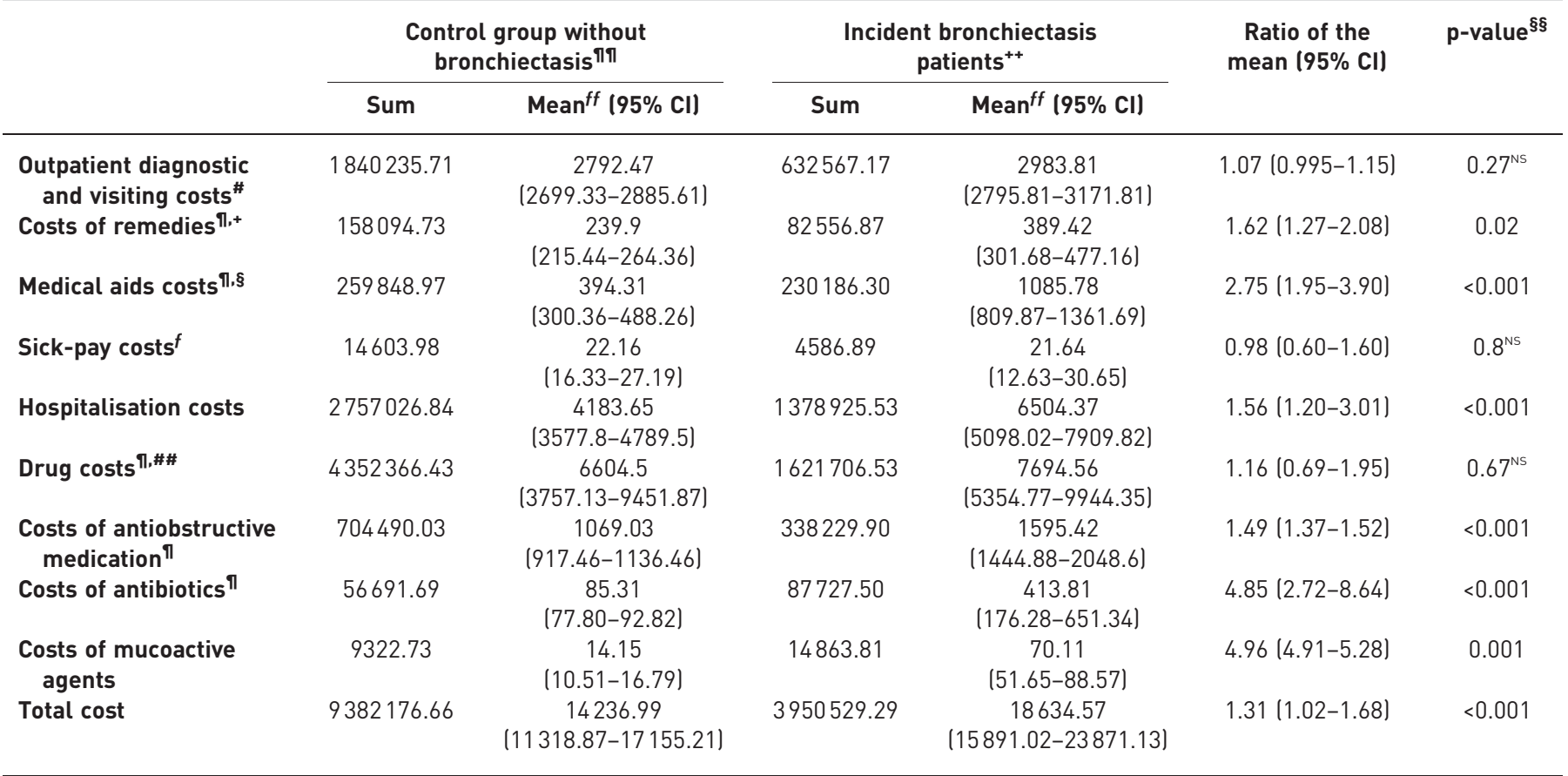

Data are presented as EUR, unless otherwise stated. "\#: outpatient costs comprise reimbursement for outpatient physician's office visits, laboratory diagnostics and imaging; ${ }^{\text {П: }}$ as outpatients; ${ }^{+}$: remedies comprise physiotherapy treatments and modalities such as active cycle of breathing and postural drainage techniques; ${ }^{\S}$ : medical aids comprise nebulisers and respiration therapy equipment; ${ }^{f}$ : sick pay is paid out in the statutory company health insurance as a substitute wage from day 43 of the sick leave according to Section 44 of the 5 th German Social Code (SGB V; www.sozialgesetzbuch-sgb.de); \#\#: prescribed pharmaceuticals according to German national drug (Anatomical Therapeutic

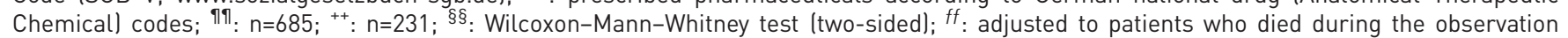
period. NS: nonsignificant.

1.02-1.68) higher than the expenditure for a matched control (EUR14236.99, 95\% CI EUR11318.77-17 155.21; $\mathrm{p}<0.001$ ) (table 3). $47.6 \%$ of the bronchiectasis patients were hospitalised at least once during follow-up and more than one-third (34.9\%) of the total cost (EUR6504.37, 95\% CI EUR5098.02-7909.82) could be attributed to costs accrued in the hospital sector. While the diagnostic and drug costs in the outpatient setting did not differ between bronchiectasis patients and matched controls, hospital costs were $56 \%$ higher in the bronchiectasis group (RoM 1.56, 95\% CI 1.20-3.01; p<0.001). It was striking that bronchiectasis patients were more often (62\%) prescribed mucoactive therapies and that the costs of those agents were nearly 5 times higher in bronchiectasis patients: EUR70.11 per bronchiectasis patient versus EUR14.15 per matched control patient (RoM 4.96, 95\% CI 4.91-5.28; $\mathrm{p}=0.001$ ). In addition, the costs of medical aids, especially inhalation and home ventilation devices, were nearly 3 times higher in bronchiectasis patients (RoM 2.75, 95\% CI 1.95-3.90; $\mathrm{p}<0.001$ ) (table 3). On average, bronchiectasis patients spent 4.85 (95\% CI 2.27-7.43) more days in hospital during the observation period (table 4).

The major part of the direct total costs, however, related to drug costs in the outpatient setting, accumulating to EUR7694.56 (95\% CI EUR5354.77-9944.35), which accounted for 41.3\% of total direct costs. On average, the frequency of visits per bronchiectasis patient to a chest physician was $83 \%$ higher (2.51, 95\% CI 2.34-2.90 versus $1.37,95 \%$ CI $1.24-1.50 ; \mathrm{p}<0.001)$ and visits to a radiologist were also $28 \%$ higher $(2.89,95 \%$ CI $2.57-3.03$ versus $2.26,95 \%$ CI $2.12-2.35 ; \mathrm{p}<0.001)$ within the 3 -year follow-up period (table 4).

\section{Antibiotic treatment}

Of the 231 patients with newly diagnosed bronchiectasis, $88.2 \%$ were prescribed any type of antibiotics in the outpatient setting during the entire 3 -year follow-up period, but only $59.7 \%$ patients of the matched control group $(p<0.001)$. In total, costs of antibiotics amounted to EUR87728 for bronchiectasis outpatients and EUR56219 for matched control patients. Out of a total of 861 prescriptions, fluoroquinolones were the most prescribed antibiotics in bronchiectasis patients (326 prescriptions (37.9\%)), followed by aminopenicillin (187 prescriptions $(21.7 \%)$ ) and macrolides (90 prescriptions (10.5\%)). 
TABLE 4 Disease-related event numbers for bronchiectasis patients compared with matched control patients

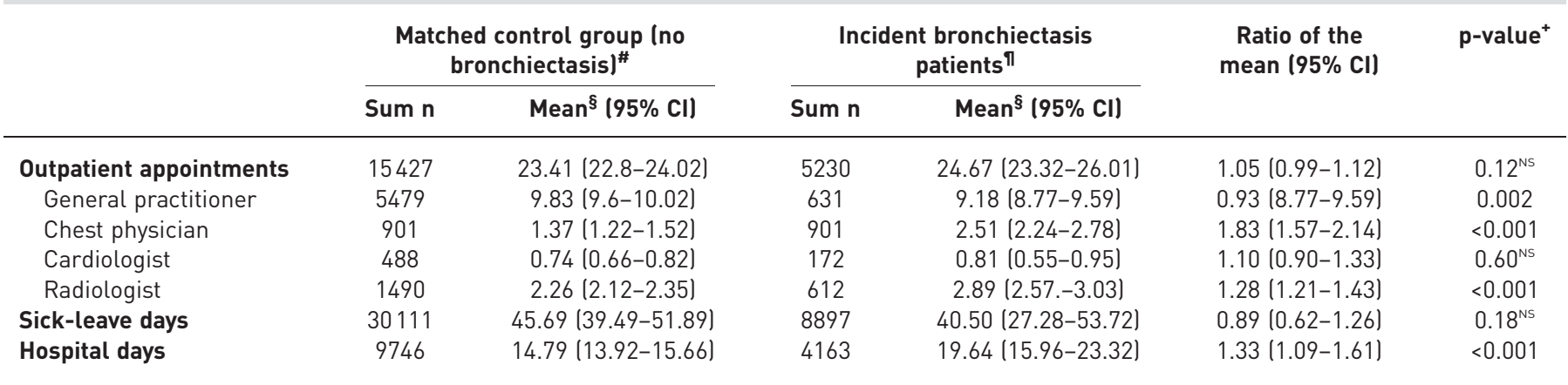

\#: $n=685 ;$ १: $n=231 ;{ }^{+}$: Wilcoxon-Mann-Whitney test (two-sided); ${ }^{\S}$ : adjusted to patients who died during the observation period. Ns: nonsignificant.

Overall, only eight bronchiectasis patients $(0.35 \%)$ and none of the matched control patients received inhaled antibiotics (all colistin) during the observation period. 13 out of the 231 patients (5.6\%) received monotherapy with clarithromycin or azithromycin for at least two subsequent quarters, indicating that this therapy was administered for maintenance treatment of bronchiectasis rather than for treating other respiratory infections. Of note, parenteral antibiotics (e.g. amikacin, ceftazidime or imipenem) were not prescribed for any bronchiectasis patient in the outpatient setting.

\section{Treatment with bronchodilators}

Costs of inhaled bronchodilators (ATC code R03) per bronchiectasis patient, of which salbutamol and long-acting $\beta_{2}$-agonists, such as salmeterol and formoterol, had particularly often been prescribed, were nearly 50\% (RoM 1.49, 95\% CI 1.37-1.52) higher than in the matched controls (EUR1595.42, 95\% CI EUR1444.992048.6 versus EUR1069.03, 95\% CI EUR917.46-1136.46). 82.1\% of bronchiectasis patients, but only 56.3\% of the matched controls, claimed at least one R03 prescription during the 3-year follow-up period.

\section{Treatment with other drugs}

As expected, costs of mucoactive drugs during the observation period were significantly higher (nearly 5 times) at EUR70.11 per bronchiectasis patient versus EUR14.15 per matched control patient (RoM 4.96, 95\% CI 4.95-4.98; $\mathrm{p}<0.001)$ and costs of topical nasal corticosteroids were nearly 4 times higher at EUR21.57 versus 6.06 (RoM 3.56, 95\% CI 3.55-3.58; $<<0.001$ ).

\section{Ranking of drugs prescribed for bronchiectasis patients and matched controls}

As shown in table 5, drug prescriptions in both groups focused on long-acting $\beta_{2}$-agonists, used with or without inhaled steroids, and the antibiotics amoxicillin, ciprofloxacin and cefuroxime axetil. Significant differences between bronchiectasis patients and their matched controls could only be found with respect to the use of azithromycin and aclidinium bromide, the latter being a long-acting muscarinic agent which before 2012 had not been approved in the European Union for treating COPD.

\section{Mortality}

Differences in all-cause mortality rates per year between the three groups of bronchiectasis patients with COPD, bronchiectasis patients without COPD and matched controls are shown in table 6.61 of the initial 231 diagnosed patients in the bronchiectasis group (26.41\%), but only 72 of the initial 651 patients in the matched control group (10.5\%), died within the 3-year follow-up period ( $\mathrm{p}<0.0001$, log-rank test) (table 6). When using Cox's regression and only adjusting for disease group, bronchiectasis patients had a nearly 4-fold higher risk of death (HR 3.64, 95\% CI 2.28-5.77; $\mathrm{p}<0.0001$ ). When adjusting for age, sex and the presence of COPD as comorbidity, sex and the presence of COPD had no significant influence on the hazard ratio, while bronchiectasis patients had a relative $5.8 \%$ increase in mortality rate per life-year compared with the matched controls.

However, when the association between COPD and mortality in the bronchiectasis group was considered individually, mortality in the 128 patients with COPD was significantly higher than in those without COPD (35.9\% (46 out of 128 ) versus $14.6 \%$ (15 out of 103 ); $\mathrm{p}<0.001$ ). 
TABLE 5 Top 25 prescribed drugs for bronchiectasis patients compared with matched control patients

\begin{tabular}{|c|c|c|c|c|c|c|c|c|c|c|}
\hline \multirow[t]{2}{*}{ ATC code } & \multirow[t]{2}{*}{ Agent } & \multicolumn{4}{|c|}{ Bronchiectasis patients } & \multicolumn{4}{|c|}{ Matched control patient } & \multirow[t]{2}{*}{ p-value ${ }^{\#}$} \\
\hline & & $\begin{array}{c}\text { Prescriptions } \\
\%\end{array}$ & $\begin{array}{c}\text { Prescriptions } \\
\mathrm{n}\end{array}$ & Rank & $\begin{array}{l}\text { Prescriptions per } \\
\text { patient } \mathrm{n}\end{array}$ & $\begin{array}{c}\text { Prescriptions } \\
\%\end{array}$ & $\begin{array}{c}\text { Prescriptions } \\
\mathbf{n}\end{array}$ & Rank & $\begin{array}{l}\text { Prescriptions per } \\
\text { patient }\end{array}$ & \\
\hline R03ACO2 & Salbutamol & 30.66 & 449 & 1 & 6.91 & 32.02 & 1.396 & 1 & 6.62 & $0.75^{\mathrm{NS}}$ \\
\hline R03AK07 & Formoterol/budesonide & 22.64 & 334 & 2 & 6.96 & 18.21 & 849 & 4 & 7.08 & $0.96^{\mathrm{NS}}$ \\
\hline J01CA04 & Amoxicillin & 21.23 & 95 & 3 & 2.11 & 18.66 & 266 & 3 & 2.16 & $0.82^{\mathrm{NS}}$ \\
\hline J01MA02 & Ciprofloxacin & 20.75 & 105 & 4 & 2.39 & 16.24 & 208 & 5 & 1.94 & $0.91^{\mathrm{NS}}$ \\
\hline R03BB04 & Tiotropium bromide & 19.34 & 342 & 5 & 8.34 & 13.51 & 605 & 7 & 6.80 & $0.82^{\mathrm{NS}}$ \\
\hline J01DC02 & Cefuroxime & 17.92 & 117 & 6 & 3.08 & 27.01 & 329 & 2 & 1.85 & $0.67^{\mathrm{NS}}$ \\
\hline J01CR22 & Amoxicillin/clavulanic acid & 16.51 & 92 & 7 & 2.63 & 8.80 & 103 & 13 & 1.78 & $0.45^{\mathrm{NS}}$ \\
\hline J01FA10 & Azithromycin & 15.57 & 87 & 8 & 2.64 & 8.65 & 117 & 14 & 2.05 & 0.004 \\
\hline R03AK06 & Salmeterol/fluticasone & 14.62 & 257 & 9 & 8.29 & 13.20 & 599 & 9 & 6.89 & $0.66^{\mathrm{NS}}$ \\
\hline R03BA02 & Budesonide & 14.15 & 176 & 10 & 5.87 & 10.62 & 355 & 10 & 5.07 & $0.85^{\mathrm{NS}}$ \\
\hline J01FA09 & Clarithromycin & 12.74 & 57 & 11 & 2.11 & 6.37 & 117 & 16 & 2.79 & $0.05^{\mathrm{NS}}$ \\
\hline J01AA02 & Doxycycline & 12.26 & 52 & 12 & 2.00 & 13.35 & 179 & 8 & 2.03 & $0.73^{\mathrm{NS}}$ \\
\hline R03AC13 & Formoterol & 11.32 & 181 & 13 & 7.54 & 14.57 & 646 & 6 & 6.73 & $0.47^{\mathrm{NS}}$ \\
\hline R01AD09 & Mometasone & 10.85 & 112 & 14 & 4.87 & 5.31 & 120 & 19 & 3.43 & $0.41^{\mathrm{NS}}$ \\
\hline R05CB01 & Acetylcysteine & 10.38 & 133 & 15 & 6.05 & 4.86 & 120 & 21 & 3.75 & $0.45^{\mathrm{NS}}$ \\
\hline R03AK03 & $\begin{array}{l}\text { Ipratropium bromide/ } \\
\text { fenoterol }\end{array}$ & 9.91 & 141 & 16 & 6.71 & 5.92 & 316 & 18 & 8.10 & $0.89^{\mathrm{NS}}$ \\
\hline J01MA12 & Levofloxacin & 9.43 & 91 & 17 & 4.55 & 9.41 & 120 & 12 & 1.94 & $0.57^{\mathrm{NS}}$ \\
\hline J01MA14 & Moxifloxacin & 8.96 & 43 & 18 & 2.26 & 6.07 & 78 & 17 & 1.95 & $0.64^{\mathrm{NS}}$ \\
\hline J01FF01 & Clindamycin & 8.02 & 32 & 19 & 1.88 & 10.32 & 139 & 11 & 2.04 & $0.89^{\mathrm{NS}}$ \\
\hline J01FA06 & Roxithromycin & 8.02 & 24 & 19 & 1.41 & 8.19 & 113 & 15 & 2.09 & $1.0^{\mathrm{NS}}$ \\
\hline R03DA04 & Theophylline & 6.60 & 96 & 21 & 6.86 & 4.10 & 278 & 24 & 10.30 & $0.60^{\mathrm{NS}}$ \\
\hline J01CE02 & Phenoxymethylpenicillin & 6.60 & 28 & 21 & 2.00 & 5.16 & 63 & 20 & 1.85 & $0.23^{\mathrm{NS}}$ \\
\hline J01EE01 & $\begin{array}{l}\text { Sulfamethoxazole/ } \\
\text { trimethoprim }\end{array}$ & 6.60 & 38 & 21 & 2.71 & 4.25 & 64 & 23 & 2.29 & $0.09^{\mathrm{NS}}$ \\
\hline R03BB05 & Aclidinium & 6.13 & 48 & 24 & 3.69 & 1.82 & 65 & 26 & 5.42 & 0.002 \\
\hline R03BB01 & Ipratropium bromide & 6.13 & 72 & 24 & 5.54 & 2.28 & 70 & 25 & 4.67 & $0.83^{\mathrm{NS}}$ \\
\hline
\end{tabular}




\begin{tabular}{lccc} 
TABLE 6 Unadjusted mortality rates by follow-up years & & \\
& Patients at risk $\mathbf{n}$ & Patients diseased $\mathbf{~}$ & Mortality $\%$ \\
\hline Matched controls & & & \\
$\quad$ Year 1 & 685 & 26 & 3.80 \\
Year 2 & 659 & 27 & 4.10 \\
Year 3 & 632 & 19 & 3.01 \\
Total & 685 & 72 & 10.51 \\
Bronchiectasis & & & \\
Year 1 & 231 & 19 & 8.23 \\
Year 2 & 212 & 25 & 11.79 \\
Year 3 & 187 & 17 & 9.09 \\
Total & 231 & 61 & 26.41 \\
Bronchiectasis without COPD & & & 0.0 \\
$\quad$ Year 1 & 103 & 0 & 5.83 \\
Year 2 & 103 & 6 & 9.28 \\
Year 3 & 97 & 9 & 14.56 \\
Total & 103 & 15 & \\
\hline
\end{tabular}

COPD: chronic obstructive lung disease.

Cost due to loss of productivity

Bronchiectasis patients had on average 40.5 (95\% CI 17.1-43.5) sick-leave days during the follow-up period and induced indirect costs of EUR4230.49 (95\% CI EUR2849.58-5611.20). However, for the same period, 45.7 (95\% CI 39.5-51.9) sick-leave days and a productivity loss of EUR4776.50 (95\% CI EUR4127.84-5425.15) were also found in the matched controls.

Cost due to absenteeism from work amounted to EUR1916.43 per bronchiectasis patient in the first year of follow-up and decreased slightly to EUR1273.61 by the third year. Considering that total direct medical costs of bronchiectasis patients are EUR18634.57, work-loss costs in bronchiectasis patients are $25.6 \%$ of that amount. The work absence burden for employees with bronchiectasis was not significantly different from that for matched controls.

\section{Discussion}

The objective of this study was to provide the first comprehensive estimate of the economic burden of incident bronchiectasis in any European country. This was accomplished by a retrospective observational design comparing medical and productivity-related expenditure for 231 bronchiectasis patients to a $1: 3$ matched comparison group of 685 control patients without bronchiectasis over a period of 3 years in Germany. The economic burden attributed to bronchiectasis is expected to be great, because it is a chronic disease that may require frequent medical consultations, long-term treatment with multimodal regimens and hospitalisations for pulmonary exacerbations in order to minimise the risk of further progression. However, to date, published annual cost data for the treatment of bronchiectasis are sparse.

The studies of SAnChez-Munoz et al. [17] and DE LA Rosa et al. [18], which retrospectively determined the hospital costs of bronchiectasis in Spain (cost year 2013), reported mean costs of EUR4672 \pm 6281 per patient [17] or EUR3515 for patients with bronchiectasis as a primary diagnosis and EUR4559 for patients with a secondary diagnosis [18]. Three North American studies calculated the annual costs of prevalent bronchiectasis patients ranging from USD13244 (cost year 2001) [19] to over USD37 030 (in patients with exacerbation, 2008-2011) [20] and up to USD67 764 (in patients with P. aeruginosa, 2007-2013) [21].

With respect to incident bronchiectasis patients, only the study of JoIsH et al. [20] examined the increase in resource use and costs for patients with bronchiectasis before and after the first year of diagnosis. US MarketScan Research data were analysed for the period from 2005 to 2009 for patients diagnosed with bronchiectasis $(n=9146)$ and controls without bronchiectasis $(n=27438)$ who were matched to each bronchiectasis patient based on age, sex, geographical region and type of health plan enrolled in a 3:1 ratio. This resulted in an increase of USD2319 per bronchiectasis patient in the first year after diagnosis, but of only USD1607 for control patients. However, in contrast to our study, where COPD as one of the most important associated conditions of bronchiectasis was explicitly allowed in both bronchiectasis cases and matched control patients, individuals with COPD were excluded if the claim was either 12 months prior (baseline) or during the subsequent 12-month follow-up period.

To the best of our knowledge, our pilot study represents the first investigation of healthcare resource use and costs brought about by incident bronchiectasis cases worldwide who were matched according to type 
and severity of their comorbidities, and for which accumulated post-diagnosis costs have been followed for several years. Furthermore, it includes (for the first time) both mortality and costs due to lost patient productivity.

When considering only respiratory-related resource utilisation, bronchiectasis outpatients sought specialised respiratory care $80 \%$ more often and were referred to radiologists $30 \%$ more often (table 4 ). Based on records for the 25 most prescribed drugs for both groups, we found no notable difference between the two groups in prescription patterns for antibiotics addressing exacerbations (fluoroquinolones, aminopenicillins and macrolides) or for bronchodilators (predominantly salbutamol and long-acting $\beta_{2}$-agonists) (table 5). In total, however, bronchiectasis patients received prescriptions for bronchodilators and antibiotics $27 \%$ and $29 \%$ more frequently, respectively, than was the case with matched controls (all $\mathrm{p}<0.001)$.

Beyond the expected economic burden on the healthcare system, mortality in our bronchiectasis group after 3 years of follow-up was surprisingly high at 26.4\%. Although in Cox's regression the presence of COPD had no significant influence on mortality when comparing the bronchiectasis group and the matched control group in which a considerable proportion of patients (35.9\%) also had COPD, mortality in the bronchiectasis group was significantly higher in patients with COPD than in those without COPD. This is in line with recently published evidence that mortality may be higher among those who also have COPD than in patients with bronchiectasis alone [22]. Because mortality in the bronchiectasis group was highest at $8.23 \%$ (95\% CI 5.33-12.49\%) within the first year following diagnosis, immediate and appropriate medical treatment should be offered as complications may arise.

Apart from the contribution of COPD, we were not able to investigate the reasons for the high mortality found among bronchiectasis patients. It may, however, be speculated that high-resolution computed tomography, the radiological investigation of choice (regular chest radiography being generally insensitive to the changes caused by bronchiectasis), was performed too late. Thus, a considerable fraction of bronchiectasis patients may have been chronically infected with bacterial pathogens well before the diagnosis of bronchiectasis was made. Unfortunately, we could not evaluate P. aeruginosa infection in our study sample as ICD-10 coding of bacterial pathogens is voluntary in Germany and therefore not routinely performed by physicians in the outpatient setting where $>90 \%$ of bronchiectasis patients are cared for [9]. According to data from the representative German Bronchiectasis Registry PROGNOSIS (The PROspective German NOn-CF bronchiectaSIS patient registry), which is part of the European Bronchiectasis Initiative EMBARC (www.bronchiectasis.eu) and which currently has recruited more than 1000 patients, $P$. aeruginosa is the most prevalent pathogen during stable disease and during pulmonary exacerbations, with detection rates of $30 \%$ and $36 \%$, respectively [23]. A high proportion of bronchiectasis patients (40.3\% (93 out of 231)) were hospitalised at least once in the first year following diagnosis. This observation suggests that the sicker patients whose disease was already fairly advanced at diagnosis may have started treatment with medication that proved at least partly ineffective with regard to the prevention of severe exacerbations requiring hospitalisations. This may have contributed to the poor prognosis of these patients within the follow-up period.

We also took into account reduced productivity caused by ongoing symptoms and exacerbations of bronchiectasis, which amounted to EUR1916.43 per bronchiectasis patient in the first year of follow-up and decreased slightly to EUR1273.61 in the third year. As the total cost of EUR4230.39 per bronchiectasis patient due to absenteeism from work that occurred within the 3 years of observation accounted for $22.7 \%$ of the burden caused by direct total costs, indirect costs may be considered to have a substantial economic impact on healthcare. Because short-term disability with sick leave of $<3$ days is not captured by the statutory company health insurance, these costs are most certainly underestimated in our calculations. The importance of indirect costs in bronchiectasis patients is not diminished by the fact that these did not differ significantly from the indirect costs in the matched control group, which was characterised not only by an equally high percentage of COPD (35.9\%), but also by a comparable prevalence of cardiac comorbidities and asthma.

When extrapolating our age- and sex-adjusted incidence rate for bronchiectasis of 6.1 (95\% CI 6.0-6.3) per 100000 insured German inhabitants and keeping this incidence rate constant, on average a total of 5045 (range 4962-5210) newly diagnosed bronchiectasis patients per year can be expected in the 2017 German population of 82.7 million [24], whereby males aged $>65$ years, probably due to a high rate of concomitant COPD, are the most strongly represented bronchiectasis subpopulation. The annual direct and indirect expenditures attributable to the disease can be expected to amount to more than EUR38.45 million per year (range EUR37.82-39.71 million per year).

Our analysis was limited by several factors. First, we only followed bronchiectasis patients for 3 years after the quarter when the diagnosis was established and thus did not capture the long-term burden of the 
disease. Accordingly, future studies of the long-term costs of bronchiectasis that also take the burden arising from prevalent cases into account are warranted. Second, other costly comorbid conditions that were not accounted for via the CCI in the matching process may have influenced the cost burden estimates. Third, beyond determining the difference of costs with respect to the presence or absence of bronchiectasis, matching on the basis of an equivalent CCI score level does not allow the costs of COPD or other diseases included in the CCI to be determined separately.

Fourth, although the number of insured persons from which bronchiectasis patients and their matched controls were taken was large at more than 4.8 million, our patients were not formally selected as part of a representative sample. Furthermore, our higher proportion of males with bronchiectasis, a disease considered more common in females than in males in Western countries [25, 26], may reflect the generally higher proportion of insured males in all age groups of our study population, which in 2012 included in total 614744 more males than females in German company health insurance funds [27]. Thus, it is not certain whether our results may be generalised to the entire German population. In addition, patients were only included in our analysis if they had no diagnosis of bronchiectasis within the 3 years prior to the coding date in 2012 and if they were continuously eligible for comprehensive health benefits in the 3 years following the diagnosis. Consequently, the figure of 6.1 newly diagnosed bronchiectasis patients per 100000 insured persons in our sample is lower than those estimated in the recently published studies of QUINT et al. [10] and WEYCKER et al. [11], with 21.2 and 29 incident cases per 100000 person-years in the UK and USA, respectively.

Nevertheless, our results not only demonstrate that per-patient spending on bronchiectasis is high, but also suggest that efforts at providing earlier and more effective treatment are warranted with a view towards lowering both mortality and costs. Indeed, although the present findings have to be seen in the context of the German healthcare system, we expect that studies subsequently performed in other Western countries would arrive at similar economic conclusions. However, although European guidelines have recently been published [25], they have not yet been implemented in Germany. National guidelines are desperately needed given the drastic risk of refund claims for costly off-label prescriptions from statutory health insurance companies, as indicated by the infrequent use of inhaled antibiotics in our "real-life" study sample.

\section{Conclusions}

Although bronchiectasis is considered to be underdiagnosed, the mortality and the associated financial burden in Germany are substantial. Efforts to manage bronchiectasis costs may be directed at reducing hospitalisation expenditures, which are the main cost drivers. Providing early and effective therapeutic interventions that can prevent disease progression may further reduce the associated economic burden of bronchiectasis.

Conflict of interest: R. Diel reports grants from Bayer Vital, during the conduct of the study; personal fees for advisory board work, consultancy and lectures from Insmed Inc., personal fees for advisory board work and consultancy from Bayer Vital, outside the submitted work. J.D. Chalmers reports research grants and personal fees from GSK, Boehringer Ingelheim, Pfizer, Bayer Healthcare, Grifols and Insmed, grants from AstraZeneca, and personal fees for consultancy from Napp and Aradigm Corporation, outside the submitted work; and is Deputy Chief Editor of the European Respiratory Journal. K.F. Rabe has nothing to disclose. A. Nienhaus has nothing to disclose. R. Loddenkemper has nothing to disclose. F.C. Ringshausen reports research support, fees for consulting, lecture fees and support of patient educational events from Bayer Healthcare, research support and consultancy and lecture fees from Grifols Germany, research support, fees for consulting, clinical trial participation and lecture fees from Insmed Germany, personal fees for lecturing and consulting from AstraZeneca, travel reimbursement, support of patient educational events and clinical trial participation from Chiesi, support of patient educational events from Abbott, Pfizer, Oxycare, Heinen+Löwenstein, MSD, PARI and APOSAN, travel reimbursement and support of patient educational events from Gilead, lecture fees, research support (IMI/EU), clinical trial participation and support of patient educational events from Novartis, research support and support of patient educational events from InfectoPharm, clinical trial participation for Vertex, Parion, Celtaxsys, Corbus, Zambon and GSK, research support (IMI/EU) from Polyphor, and lecture fees from Boehringer Ingelheim, outside the submitted work.

Support statement: Provision of statutory company health insurance data was supported by Bayer Vital (Leverkusen, Germany) with an unrestricted grant. Bayer Vital had no role in the study design, contents and preparation of this manuscript or the decision to publish.

\section{References}

1 Hill A, Pasteur M, Cornford C, et al. Primary care summary of the British Thoracic Society Guideline on the management of non-cystic fibrosis bronchiectasis. Prim Care Respir J 2011; 20: 135-140.

2 Finch S, McDonnell MJ, Abo-Leyah H, et al. A comprehensive analysis of the impact of Pseudomonas aeruginosa colonization on prognosis in adult bronchiectasis. Ann Am Thorac Soc 2015; 12: 1602-1611.

3 King P. The pathophysiology of bronchiectasis. Int J Chron Obstruct Pulmon Dis 2009; 4: 411-419. 


3

26 McShane PJ, Naureckas ET, Tino G, et al. Non-cystic fibrosis bronchiectasis. Am J Respir Crit Care Med 2013; 188: 647-656.

27 Federal Ministry of Health. Zahlen und Fakten zur Krankenversicherung. [Facts and figures about health insurance.] 2018. www.bundesgesundheitsministerium.de/themen/krankenversicherung/zahlen-und-fakten-zur-krankenversicherung. html Date last accessed: November 19, 2018. 